\title{
Intrauterine device migration to the urinary bladder
}

\author{
Authors: $\underline{K a r l a ~ J u r i c ́}^{1}$, Ivan Putica ${ }^{1}$, Mario Ćorić ${ }^{1,2}$ (mentor) \\ ${ }^{1}$ School of Medicine, University of Zagreb, Zagreb, Croatia \\ 2 Department of Obstretics and Gynecology, Clinical Hospital Center Zagreb, Zagreb, Croatia \\ DOI: https://doi.org/10.26800/LV-142-supp5-10
}

Background:

The intrauterine device (IUD) is a common method of contraception among women. As one of complications of IUD, perforation occurs in approximately 1-2 in 1000 insertions. Although migration of IUD may pursue asymptomatic course, symptoms of migration to the urinary bladder can include lower urinary tract symptoms, stone formation around the IUD and uterovesical fistula. In this report, we present a case of an IUD migration into the urinary bladder of a 34-year-old woman at the Clinical Hospital Center Zagreb.

\section{Case presentation:}

A 34-year-old woman presented with macrohematuria in the Department of Urology. No other symptoms were present. Physical examination was normal. Urinalysis revealed macrohematuria, while other routine laboratory investigations had normal findings. She was otherwise healthy. Patient had a copper IUD placed seven years ago and became pregnant within 1 month IUD of insertion. Transabdominal ultrasound showed suspicion of concretion. MSCT was performed and malpositioned IUD in the urinary bladder was shown. Patient was referred to the Department of Obstetrics and Gynecology. Transvaginal ultrasound was performed and hyperechogenicity was shown, resembling an IUD. Cystoscopy revealed a part of an IUD. The IUD was removed through urethra using Bettocchi hysteroscope. Permanent catheter was placed for 30 days. There were no postoperative complications.

\section{Conclusion:}

The presence of macrohematuria in women with an IUD in the history should raise the suspicion of an IUD migration to the urinary bladder. Cystoscopic removal using Bettocchi hysteroscope can be considered as a safe and minimally invasive approach in managing a migrated IUD in the bladder.

Keywords: intrauterine device, macrohematuria, urinary bladder, uterine perforation 